Mehmet Akif Ersoy Üniversitesi Fen Bilimleri Enstitüsü Dergisi 10(2): 189-197 (2019)

The Journal of Graduate School of Natural and Applied Sciences of Mehmet Akif Ersoy University 10(2): $189-197$ (2019)

Araştırma Makalesi / Research Paper

\title{
2.45 GHz Frekasında Optimum Bant Durduran Filtre Tasarımı ve Optimizasyonu
}

\author{
Büşra ÖZTÜRK (D) ${ }^{\star}$, Özlem COŞKUN ${ }^{D 2} 2$ \\ ${ }^{1}$ Süleyman Demirel Üniversitesi, Fen Bilimleri Enstitüsü, Isparta \\ ²üleyman Demirel Üniversitesi, Mühendislik Fakültesi, Isparta \\ Geliş Tarihi (Received): 29.05.2019, Kabul Tarihi (Accepted): 24.11.2019 \\ $\square$ Sorumlu Yazar (Corresponding author*): busra_5426@hotmail.com \\ (C) +902462111373 圆 +902462370859
}

öz

Filtre yapılarının RF/mikrodalga uygulamalarındaki önemi oldukça fazladır. Bant durduran filtreler biyomedikal cihazlarda da sıklıkla kullanılmaktadır. Biyomedikal cihazlarda ölçüm yapılırken havada dolaşan istenmeyen sinyallerin sapmalara neden olmaması için, cihazlara bu frekanstaki sinyalleri durduran filtreler konulmaktadır.WLAN uygulamalarından olan IEEE $802.11 \mathrm{~b}$ azami $11 \mathrm{Mbit} / \mathrm{s}$ lik bir ham hıza sahiptir ve orijinal standartta aynı cihazları bağlamak için kullanılmaktadır. IEEE $802.11 \mathrm{~b}$ standartında çalışan aygıtlar, $2.45 \mathrm{GHz}$ ile çalışan diğer aygıtlardan (mikrodalga fırın, Bluetooth aygıtları, kablosuz telefonlar) dolayı bir parazitlenme yaşamaktadır. Bu çalışmanın ana hedefi; bu girişimlerin engellenmesi amacıyla, $2.45 \mathrm{GHz}$ frekansında bant durduran filtre tasarımı gerçekleştirilmiştir. Gerçekleştirilen bu bant durduran filtre tasarımları sayesinde; istenmeyen sinyaller filtrelenmiş, istenilen sinyallerin başarılı bir şekilde iletilmesi sağlanmıştır.

Anahtar Kelimeler: Iletim Hatları, Mikroşerit Filtre, Optimum Bant Durduran Filtre, ADS

\section{Design and Optimization of Microstrip Optimum Band-Stop Filter at $2.45 \mathrm{GHz}$}

\section{ABSTRACT}

The importance of filter structures in RF/microwave applications is very high. Band-stopping filters are also frequently used in biomedical devices. When measuring biomedical devices, filters that stop signals at this frequency are placed on the devices so that unwanted signals circulating in the air do not cause deviations. IEEE $802.11 \mathrm{~b}$ has a maximum speed of $11 \mathrm{mbits} / \mathrm{s}$, and is used to connect the same devices to the original standard. IEEE $802.11 \mathrm{~b}$ standard devices are experiencing interference due to other devices running at $2.45 \mathrm{GHz}$ (microwave oven, Bluetooth devices, wireless phones). The main objective of this work is; In order to prevent these interferences, band stop filter design at 2.45 $\mathrm{GHz}$ frequency was realized. Thanks to the filter designs that stop this band, unwanted signals have been filtered and the desired signals have been successfully transmitted.

Keywords: Transmission Line, Microstrip Filter, Optimum Band-Stop Filter, ADS

\section{GíRiş}

Günümüz uzak mesafe haberleşme sistemlerinde büyük bir sorun olarak görülen istenmeyen sinyaller (gürültü), bu sinyalleri bastırmak, iletilmesi istenen sinyalin havada dolanan sinyaller ile karışmasını engellemek amacı ile mikroldalga filtre yapıları önem arz etmektedir. Bu çaIışma ile özellikle uzak mesafe haberleşmelerinde karşılaşılan bu sorunu çözmek hedeflenmiştir. 
Mikrodalga filtrelerin RF ve mikrodalga uygulamalarında ki önemi çok büyüktür. Birbirinden farklı frekansları birleştirir veya ayırırlar. Filtre yapıları mikrodalga sistemlerinde, özellikle de uydu ve mobil haberleşme sistemlerinde sıkça kullanılmaktadır. Bu filtre yapılarında; yüksek performans, düşük kayıp, küçük boyut ve düşük maliyet gereksinimleri aranmaktadır. Genel olarak osilatör ve mikser gibi cihazlarda; istenmeyen sinyalleri engellemek için, bant durduran filtreler yapılara eklenmektedir. Bunlar gibi birçok mikrodalga sistemleri bant durduran filtre yapıları içermektedir. Bu bağlamda oluşan gereksinimleri karşılamak adına filtreler toplu eleman ve ayrık eleman devreleri olarak tasarlanır. Bu tasarımlar dalga kılavuzu, koaksiyel hat ve mikroşerit iletim hatlarından oluşmaktadır.

Günümüzde kullanılan filtreler şu an intiyacı karşılıyor da olsa tam manasıyla filtre karakteristiklerinde kazanç ve geri dönüş kaybı istenilen seviyede değildir. Bu da eldeki filtrelerin daima daha iyi olmasını gerektirmektedir. Yaptığımız bu çalışma ile litaratürden farklı olarak 3 farklı "Optimum Bant Durduran Filtre" tasarımı yapılmış olup her biri için birden fazla parametre göz önünde bulundurularak analizler yapılmış ve kendi içlerinde değerlendirilmişlerdir. Bu tasarımları yapmaktaki asıl amacımız daha yüksek performans, daha düşük geri dönüş kaybına sahip ve daha kompakt ve kullanılabilir boyutlarda bant durduran filtre tasarlamaktır.

Literatürde mikroşerit hatlar ile tasarlanmış birçok çaIışma vardır.

Hsieh ve ark. (2005) yılında yaptıkları çalışmalarında kompakt boyutlu geniş bant genişliği ve düşük ekleme kaybı özelliklerini sunan yeni bir tek kesitli bant durdurucu filtre üretmişlerdir. Bu bant durdurucu filtre, bir ucunda kısa devre olan bir bölüm anti-couple hatlı tek bir çeyrek dalga boylu rezonatör kullanılarak yapılandırımıştır. Bu tip bant durdurucu filtrelerin zayıflatma-kutup özellikleri TEM iletim hattı modeli kullanılarak araştırılmıştır. Simülasyon sonuçları ve deneysel sonuçlar arasında iyi bir uyum oluşturmuşlardır (Hsieh ve Wang, 2009).

Tu ve Chang (2006) yılında yaptıkları çalışmalarında bant durdurma filtrelerinin bant geçişi veya bant geçiren filtrelere entegrasyonunu sunmuşlardır. Hat üzerindeki geleneksel çeyrek dalga boylu saplamaları T şeklindeki hatlarla değiştirmişlerdir. İletim hattı modeli hesaplamasını eşdeğer T şeklindeki hatların tasarım denklemlerini türetmek için kullanmışlardır. Daha sonra tasarım ve ölçümler yapmışlardır (Tu ve Chang, 2006).

Shaman ve ark. (2007) yılında yaptıkları çalışmalarında çapraz bağlanmış mikroşerit bant durduran filtre için ge- nel bir devre konfigürasyonu önermişlerdir. $4 \mathrm{GHz}$ merkez frekansında yaklaşık olarak $\% 80$ bant genişliği gözlemlemişlerdir. Önerdikleri filtre yapısı teorik olarak başarıyla gerçekleştirilmiş simülasyon ve deneylerle doğrulanmıştır (Shaman ve Hong, 2007).

Habibi ve ark. (2012) yııında yaptıkları çalışmalarında $X$ bantta kullanılmak üzere geniş bantlı bir bant durdurma filtresi tasarlamışlardır. Tasarımlarını mikroşerit hat üzerine 5 çift $L$ şeklinde saplamalar yaparak oluşturmuşlardır. Oluşturulan filtrenin çalışma frekansı $L$ şeklindeki saplamaların boyutlarını değiştirerek kontrol etmişlerdir. Simülasyon sonuçları ve deneysel sonuçları mükemmel uyum göstermiştir (Habibi ve ark.,2012).

BalaSenthilMurugan ve ark. (2012) yılında yaptıkları çaIışmalarında WLAN uygulamalarında kullanılmak üzere $L$ şekilli rezonatör yapıları kullanarak bant durdurucu filtre tasarlamışlardır. Tasarımlarını $2.45 \mathrm{GHz}$ frekansında yapmışlardır ve $-60 \mathrm{~dB}$ zayıflama elde etmişlerdir. Tasarımlarını FR4 malzemesi kullanarak ADS (Gelişmiş Tasarım Sistemi) programında simüle etmişlerdir (BalaSenthilMurugan ve ark., 2012).

Yang (2015) yılında yaptığı çalışmasında kompakt çift bantta çalışan mikroşerit bant durdurucu filtre tasarımı yapmıştır. Tasarımında açık devre saplamalı yapı kullanmıştır. Tasarımında dielektrik sabiti 9.9 olan Rogers TMM10i substrat malzemesini kullanmıştır. İletim hatları boyutlarını AppCAD programı yardımıyla hesaplamıştır. ABCD matrisi analizi ile T-şekilli hatların parametrelerini hesaplamıştır. İki adet $T$ şekilli yapıyı birleştirerek çift bant Tşekilli yapı oluşturarak $2.0 \mathrm{GHz}$ ve $3.0 \mathrm{GHz}$ merkez frekanslarında çift bantta çalışan bant durdurucu filtre tasarlamıştır (Yang, 2015).

Liu ve ark. (2017) yılında yaptıkları çalışmalarında iletim hattına $\mathrm{L}$ şeklinde bağlanmış mikroşerit rezonatörlere dayanan bir dar bant durdurma filtresi tasarlamışlardır. Gevşek biçimde bağlanmış üç mikroşerit rezonatör bant durdurma filtre devresi oluşturmak için kademeli bir şekilde bağlamışlardır. Devrenin boyutunu etkili bir şekilde azaltmak için açık bir radyal yama kullanmışlardır. Tasarlanan devreyi Rogers RO4350B substrat malzeme üzerinde üretmişlerdir. Sonuç olarak üç aşamalı rezonatörlü dar bant emici bir bant durdurma filtresinin 2.23 $\mathrm{GHz}$ merkez frekansı için tasarlamışlardır. Merkez frekansında ve $20 \mathrm{MHz}$ lik bantta geri dönüş kaybı 30 dB'den daha iyi olarak ölçmüşlerdir (Liu ve ark., 2017).

Kusama ve Isozaki (2019) yılında yaptıkları çalışmalarında kompakt ve geniş bantlı bir bant durdurma filtresi tasarlamışlardır. Filtre tasarımı yaparken ana iletim hattı üzerine çeyrek dalga boylu açık devre saplamaları eklemişlerdir. Önerdikleri yapı ile radyo frekansı sinyalinin doğru akım kaynağına ters akışını önlemek amaçlı açık 
devre saplama ile T eğilimini elde etmek olmuştur. Tasarımlarında FR4 malzemesi kullanmış ve HFSS programını kullanarak analizlerini yapmışlardır. Sonuç olarak $2.5 \mathrm{GHz}$ merkez frekansında geniş bantta çalışan bant durdurucu filtre tasarımı yapmışlardır (Kusama ve Isozaki, 2019).

\section{MATERYAL VE YÖNTEM}

\section{Bant Durduran Filtre Yapısı}

Bant durduran filtrelerde asıl amaç istenmeyen ve belirlenen frekans aralığını bastırırken kalan frekans değerlerinin iletimini sağlamaktır. Bant durduran filtrelerde durdurma bandı iki iletim bandı arası olarak tanımlanır. Durdurma bandı, bandın alçak kesim frekansı fdurdurı ile bandın yüksek kesim frekansı furdur2 arasında kalan bölgedir. Bant durduran filtrelerin iki adet iletim bandı vardır. Alçak iletim bandı 0'dan başlayarak filetı'e kadar devam ederken yüksek iletim bandı filet2'den başlayarak sonsuza kadar (analog filtreler için) devam eder. Durdurma kazanç parametresi adurdur (dB)'dir. İletim bandı kazanç parametresi ise alçak ve yüksek iletim bantları için sırasıyla ailet1 (dB) ve ailet2 (dB) olarak gösterilebilir (Şekil 1).

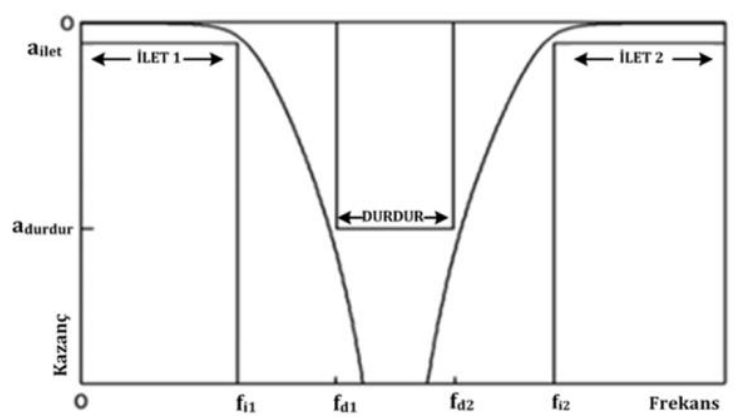

Şekil 1. Bant Durduran Filtre Frekans Cevabı

\section{Optimum Bant Durduran Filtre}

Eğer geniş bant kullanılacaksa Şekil 2.a'daki gibi açık devre saplamalar yapılması gerekir. Tasarıma birim elemanlar eklenerek, yedek birim elemanları ile tasarlanan filtreler için mümkün olan sayıdan daha fazla saplama yapılarak daha hızlı zayıflatma özellikleri elde edilebilir.

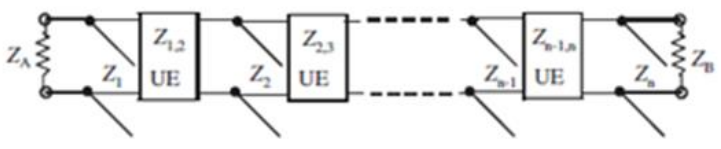

(a)

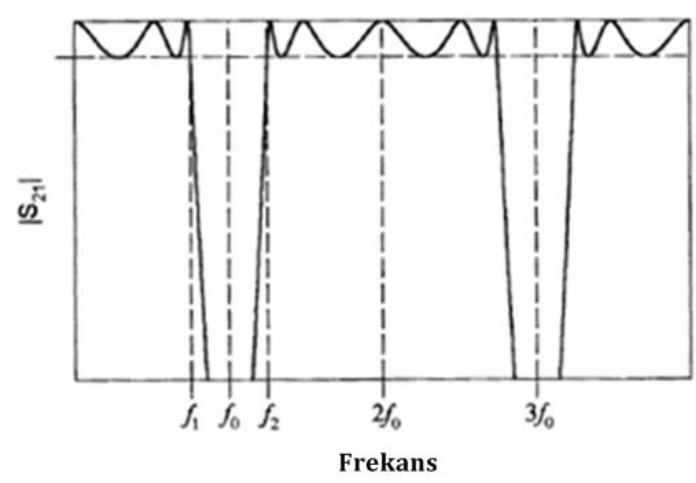

(b)

Şekil 2. a) Açık devre saplamalar içeren bant durdurucu filtrenin iletim hattı karakteristiği b) Filtrenin frekans tepkisi

$\mathrm{N}$ adet saplama ile tasarımı yapılacak olan optimum bant durduran filtre için Şekil 2.a'daki devrede ki optimum transfer fonksiyonuna bakılarak aşağıdaki gibi sentezlenir.

$$
\left|\mathrm{S}_{21}(\mathrm{f})\right|^{2}=\frac{1}{1+\varepsilon^{2} F_{N}^{2}(f)}
$$

Burada $\varepsilon$ geçiş bandı dalgalanma sabitidir ve $F_{N}$ filtreleme fonksiyonu aşağıda verilmiştir.

$$
F_{N}=T_{N}\left(\frac{1}{t_{c}}\right) T_{n-1}\left(\frac{t \sqrt{1-t_{c}^{2}}}{t_{c} \sqrt{1-t^{2}}}\right)-U_{n}\left(\frac{t}{t_{c}}\right) U_{n-1}\left(\frac{t \sqrt{1-t_{c}^{2}}}{t_{c} \sqrt{1-t^{2}}}\right) \text { (2) }
$$

Burada t Richard'ın transform değişkenidir.

$$
t=j \tan \left(\frac{\pi}{2} \frac{f}{f_{0}}\right)
$$

ve

$$
t_{c}=j \tan \left(\frac{\pi}{4}\right)(2-K B G)
$$




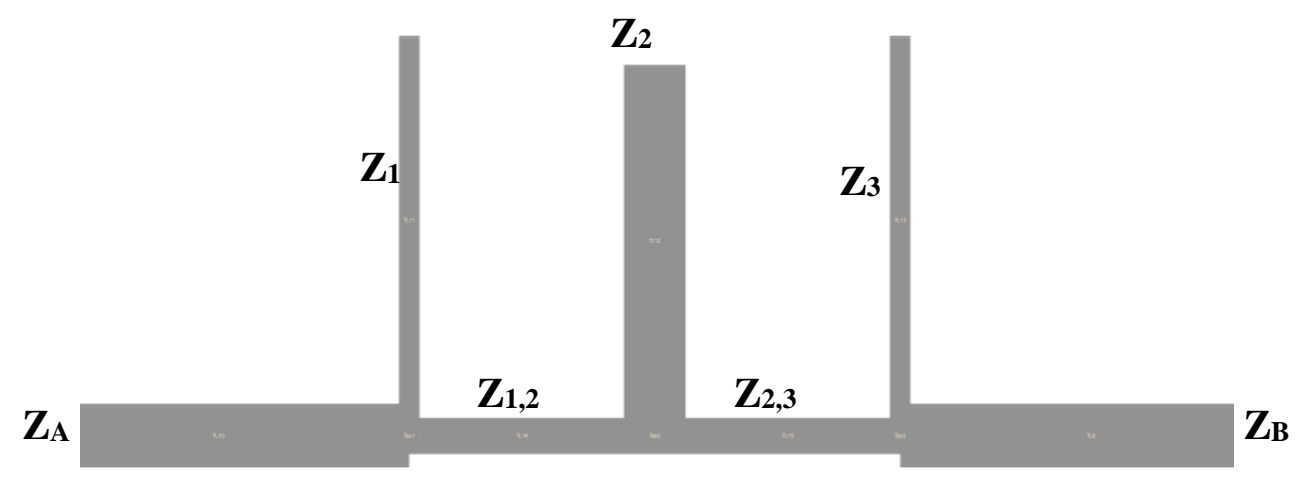

(a)

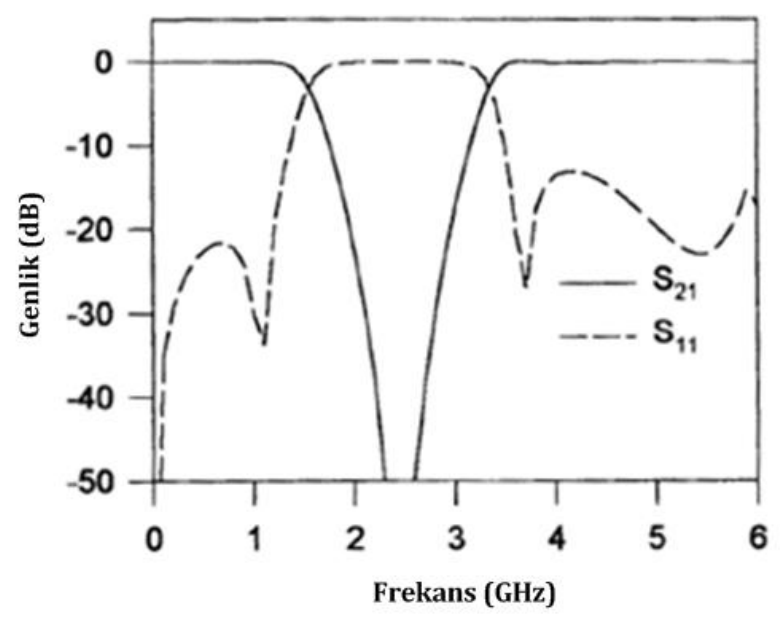

(b)

Şekil 3. a) Mikroşerit bant durdurucu filtre b) Filtrenin frekans tepkisi

Burada $f_{0}$ bant durduran filtrenin merkez frekansı olup KBG (FBW-Fractional Bandwidth) kısmi bant genişliğidir. $T_{n}(x)$ ve $U_{n}(x)$, birinci ve ikinci türdeki n'lerin Chebyshev fonksiyonlarıdır:

$$
\begin{aligned}
& T_{n}(x)=\cos \left(n \cos ^{1} x\right) \\
& U_{n}(x)=\sin \left(n \cos ^{1} x\right)
\end{aligned}
$$

Kolaylık sağlamak için, Şekil 2.a'daki devrenin eleman değerleri $\left(g_{x}, j_{x, y}\right)$ iki, üç ve dört saplamalı optimum bant durdurucu filtrelerin tasarımlarında kullanılmak üzere Tablo 1, 2 ve 3'te verilmiştir. Bu tablolardaki değerler 20 dB'lik bir geri dönüş kaybı ve $\% 30$ ile $\% 150$ kısmi bant genişlikleri için verilmiştir.
Tablo 1. $n=2$ ve $\varepsilon=0.1005$ için optimum bant durduran filtrenin eleman değerleri

\begin{tabular}{|l|l|l|}
\hline KBG (\%) & $\mathbf{g}_{\mathbf{1}}=\mathbf{g}_{\mathbf{2}}$ & $\mathbf{J}_{\mathbf{1 , 2}}$ \\
\hline 30 & 0.16989 & 0.98190 \\
\hline 40 & 0.23418 & 0.93880 \\
\hline 50 & 0.30386 & 0.89442 \\
\hline 60 & 0.38017 & 0.84857 \\
\hline 70 & 0.46470 & 0.80106 \\
\hline 80 & 0.55955 & 0.75173 \\
\hline 90 & 0.66750 & 0.70042 \\
\hline 100 & 0.79244 & 0.64700 \\
\hline 110 & 0.93992 & 0.59137 \\
\hline 120 & 1.11821 & 0.53346 \\
\hline 130 & 1.34030 & 0.47324 \\
\hline 150 & 1.62774 & 0.41077 \\
\hline & 2.01930 & 0.34615 \\
\hline
\end{tabular}


Tablo 2. $n=3$ ve $\varepsilon=0.1005$ için optimum bant durduran filtrenin eleman değerleri

\begin{tabular}{|l|l|l|l|}
\hline KBG (\%) & $\mathbf{g}_{\mathbf{1}}=\mathbf{g}_{\mathbf{3}}$ & $\mathbf{g}_{\mathbf{2}}$ & $\mathbf{J}_{\mathbf{1 , 2}}=\mathbf{J}_{\mathbf{2 , 3}}$ \\
\hline 30 & 0.16318 & 0.26768 & 0.97734 \\
\hline 40 & 0.23016 & 0.38061 & 0.92975 \\
\hline 50 & 0.37754 & 0.63292 & 0.83956 \\
\hline 60 & 0.46895 & 0.79494 & 0.78565 \\
\hline 70 & 0.56896 & 0.97488 & 0.73139 \\
\hline 80 & 0.67986 & 1.17702 & 0.67677 \\
\hline 90 & 0.80477 & 1.40708 & 0.62180 \\
\hline 100 & 0.94806 & 1.67311 & 0.56648 \\
\hline 110 & 1.11601 & 1.98667 & 0.51082 \\
\hline 120 & 1.15215 & 2.06604 & 0.49407 \\
\hline 130 & 1.37952 & 2.49473 & 0.43430 \\
\hline 140 & 1.67476 & 3.05136 & 0.37349 \\
\hline 150 & 2.07059 & 3.79862 & 0.31262 \\
\hline
\end{tabular}

Tablo 3. $n=4$ ve $\varepsilon=0.1005$ için optimum bant durduran filtrenin eleman değerleri

\begin{tabular}{|l|l|l|l|l|}
\hline $\begin{array}{l}\text { KBG } \\
(\%)\end{array}$ & $\mathbf{g}_{\mathbf{1}=\mathbf{g}_{\mathbf{4}}}$ & $\mathbf{g}_{\mathbf{2}}=\mathbf{g}_{\mathbf{3}}$ & $\mathbf{J}_{\mathbf{1 , 2}}=\mathbf{J}_{\mathbf{3 , 4}}$ & $\mathbf{J}_{\mathbf{2 , 3}}$ \\
\hline 30 & 0.23069 & 0.40393 & 0.93372 & 0.91337 \\
\hline 40 & 0.31457 & 0.55651 & 0.87752 & 0.85157 \\
\hline 50 & 0.40366 & 0.72118 & 0.82172 & 0.79093 \\
\hline 60 & 0.49941 & 0.90054 & 0.76623 & 0.73145 \\
\hline 70 & 0.60366 & 1.09802 & 0.71101 & 0.67313 \\
\hline 80 & 0.71884 & 1.31815 & 0.65598 & 0.61597 \\
\hline 90 & 0.79436 & 1.46655 & 0.62025 & 0.57951 \\
\hline 100 & 0.99642 & 1.85355 & 0.54634 & 0.50503 \\
\hline 110 & 1.10390 & 2.06672 & 0.50871 & 0.46793 \\
\hline 120 & 1.37861 & 2.59505 & 0.43702 & 0.39831 \\
\hline 130 & 1.55326 & 2.94111 & 0.39654 & 0.35972 \\
\hline 140 & 1.97310 & 3.74861 & 0.32781 & 0.29526 \\
\hline 150 & 2.43047 & 4.63442 & 0.27321 & 0.24488 \\
\hline
\end{tabular}

$\mathrm{Bu}$ tablo elemanları ve belirlenen bir referans empedansı ( $Z_{0}$ ) ile birlikte eşitlik (7), (8), (9)'da verilen empedans değerleri bulunur (Hong and Lancaster, 2001).

$$
\begin{aligned}
& Z_{A}=Z_{B}=Z_{0} \\
& Z_{i=}=Z_{0} / g_{i} \\
& Z_{i+1}=Z_{0} / J_{i, i+1}
\end{aligned}
$$

\section{ARAŞTIRMA BULGULARI}

Tasarımları yapılmış olan devrelerde substrate malzeme olarak FR4 malzemesi seçilmiştir. Bu malzemenin bağıl geçirgenlik katsayısı 4.5 kayıp tanjantı 0.022 ve $\mathrm{H}$ yüksekliği 1.6'dir. Bu tasarımların hepsinde merkez frekansı $2.45 \mathrm{GHz}$ seçilmiştir. İletim hatları $\lambda / 4$ uzunluğundadır. Mikrodalga devre analizi yapabilen bir bilgisayar destekli tasarım aracı (ADS) kullanarak saçılma parametreleri ve merkez frekansları hesaplanmıştır. Bant genişlikleri (BG) eşitlik (10) kullanılarak yüzde olarak hesaplanmıştır. $f_{2}$ ve $f_{1}$ gücün yarıya düştüğü $-3 d B$ 'de ölçülen frekans değerlerindir.

$$
\mathrm{BG}=\frac{f_{2}-f_{1}}{f_{0}} \times 100
$$

3 farklı optimum bant durduran filtre tasarımı yapılmıştır. Bu tasarımlarda kısmi bant genişliği \% 50 olarak seçilmiştir. Tasarımlarda referans empedans değeri olan $Z_{0}$ $50 \Omega$ olarak seçilmiş olup eşitlik (7)'de görüldüğü gibi $Z_{A}$ ve $Z_{B}$ değerleri de $Z_{0}$ değerine bağlı olarak $50 \Omega$ olarak hesaplanmıştır. Her bir tasarım için analizler tablolar halinde verilmiştir.

İlk olarak $n=2$ olarak seçilmiş ve buna göre Tablo 1'de bulunan değerlere göre hatların empedansları eşitlik (7),(8),(9)'a göre hesaplanmıştır.

$$
\begin{aligned}
& Z_{A}=Z_{B}=50 \Omega \\
& Z_{1}=Z_{2}=164.55 \Omega \\
& Z_{1,2}=55.9 \Omega
\end{aligned}
$$

Bu empedanslara göre hat genişlikleri ve uzunlukları hesaplanmış ve Tablo 4'te değerler verilmiştir.

Tablo 4. $n=2$ için empedansların hat genişlik ve uzunluk değerleri

\begin{tabular}{|l|l|l|}
\hline $\begin{array}{l}\text { Hat/Hat- } \\
\text { lar }\end{array}$ & $\mathbf{W}(\mathbf{m m})$ & $\left.\boldsymbol{\Lambda}_{\mathbf{g} \mathbf{0}} / \mathbf{4} \mathbf{( m m}\right)$ \\
\hline$Z_{A}, Z_{B}$ & 2.99 & 16.6 \\
\hline$Z_{1}, Z_{2}$ & 0.081 & 18.7 \\
\hline$Z_{1,2}$ & 2.46 & 16.8 \\
\hline
\end{tabular}

Daha sonra bu değerlere göre devre tasarlanmıştır. Devrenin şematiği Şekil 4'teki gibidir. S-parametre değeri Şekil 5'de gösterilmiştir. 


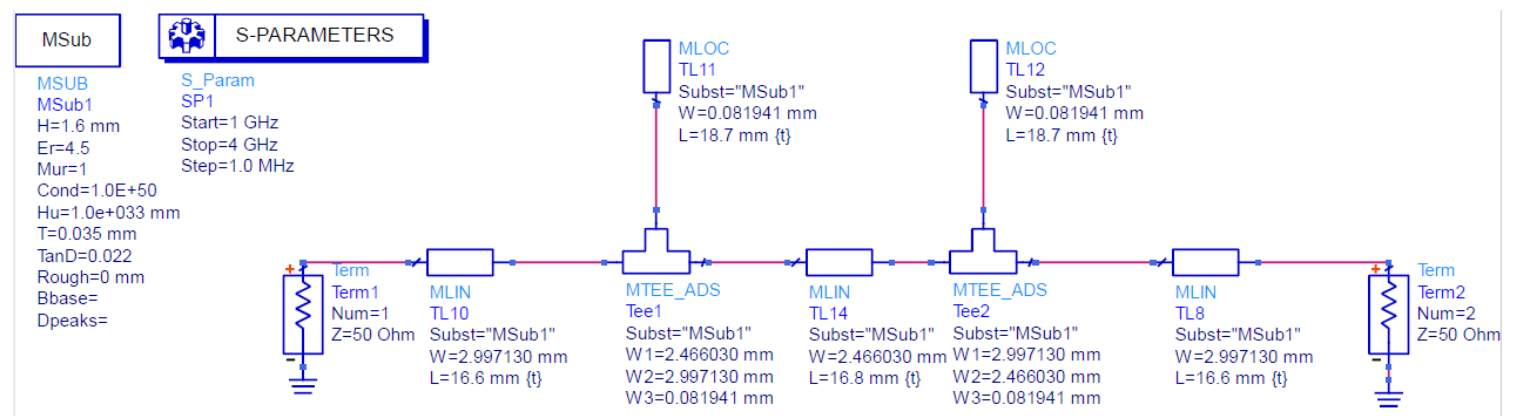

Şekil 4. Optimum bant durduran filtre tasarımı şematiği ( $n=2$ için)

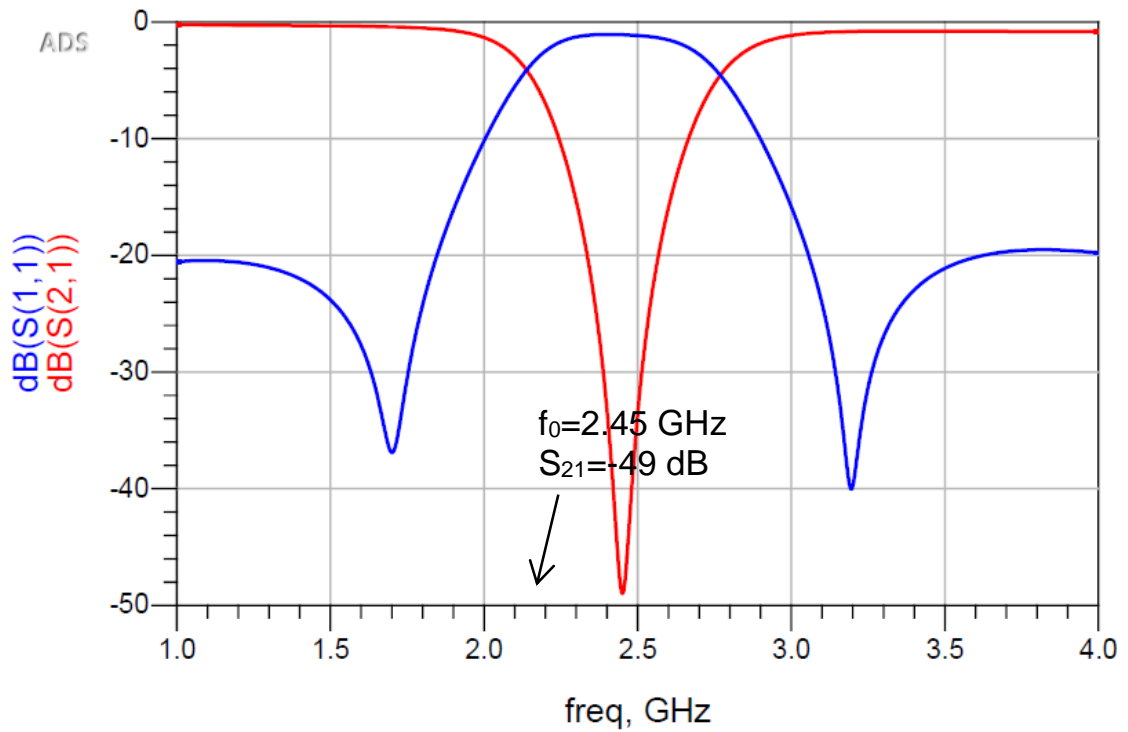

Şekil 5. Optimum bant durduran filtre $S_{11}$ ve $S_{21}$ değeri simülasyon sonucu ( $n=2$ için)

Tasarımı yapılan devrede merkez frekansını 2.45 GHz'de $S_{21}$ değeri $-49 \mathrm{~dB}$ olarak ölçülmüştür. Tasarıma ait yüzde bant genişliği eşitlik (10)'dan yararlanılarak \%29.4 olarak hesaplanmıştır.

Bir diğer tasarımda $n=3$ olarak seçilmiş ve buna göre Tablo 2'de bulunan değerlere göre hatların empedansları eşitlik (7),(8),(9)'a göre hesaplanmıştır.

$$
\begin{aligned}
& Z_{A}=Z_{B}=50 \Omega \\
& Z_{1}=Z_{3}=132.43 \Omega \\
& Z_{1,2}=Z_{2,3}=78.9 \Omega \\
& Z_{2}=59.5 \Omega
\end{aligned}
$$

Bu empedanslara göre hat genişlikleri ve uzunlukları hesaplanmış ve Tablo 5'te değerler verilmiştir.
Tablo 5. $n=3$ için empedansların hat genişlik ve uzunluk değerleri

\begin{tabular}{|l|l|l|}
\hline Hat/Hatlar & $\mathbf{W}(\mathbf{m m})$ & $\Lambda_{\mathbf{g o}} / \mathbf{4}(\mathbf{m m})$ \\
\hline$Z_{A}, Z_{B}$ & 2.99 & 16.6 \\
\hline$Z_{1}, Z_{3}$ & 0.24 & 18.1 \\
\hline$Z_{1,2}, Z_{2,3}$ & 2.2 & 17 \\
\hline$Z_{2}$ & 1.21 & 17.6 \\
\hline
\end{tabular}

Daha sonra bu değerlere göre devre tasarlanmıştır. Devrenin şematiği Şekil 6'daki gibidir. S-parametre değeri Şekil 7'de gösterilmiştir. 


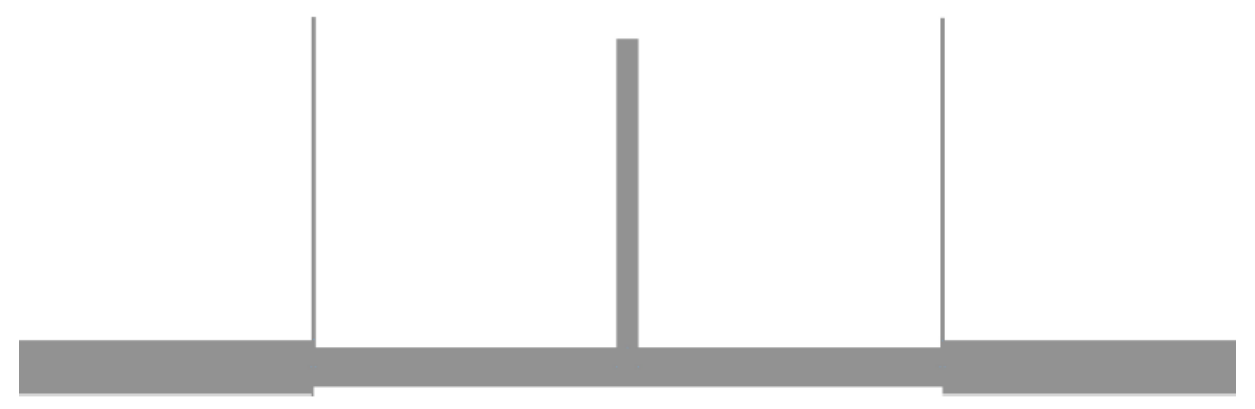

Şekil 6. Optimum bant durduran filtre tasarımı ( $n=3$ için)

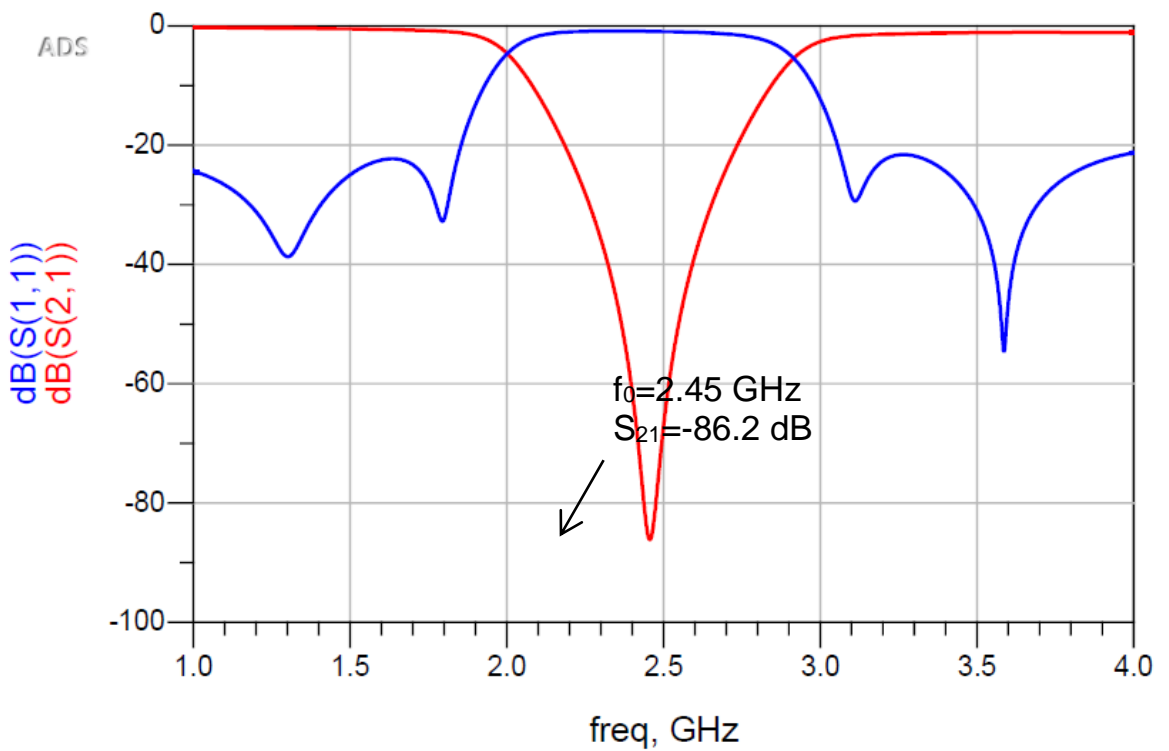

Şekil 7. Optimum bant durduran filtre $S_{11}$ ve $S_{21}$ değeri simülasyon sonucu ( $n=3$ için)

Tasarımı yapılan devrede merkez frekansı $2.45 \mathrm{GHz}$ 'de $S_{21}$ değeri -86.2 dB olarak ölçülmüştür. Tasarıma ait yüzde bant genişliği eşitlik (10)'dan yararlanılarak \%41.6 olarak hesaplanmıştır.

Son olarak tasarımda $n=4$ olarak seçilmiş ve buna göre Tablo 3'te bulunan değerlere göre hatların empedansları eşitlik (7),(8),(9)'a göre hesaplanmıştır.

$$
\begin{aligned}
& Z_{A}=Z_{B}=50 \Omega \\
& Z_{1}=Z_{4}=123.9 \Omega \\
& Z_{2}=Z_{3}=69.3 \Omega \\
& Z_{1,2}=Z_{3,4}=60.85 \Omega \\
& Z_{2,3}=63.2 \Omega
\end{aligned}
$$

Bu empedanslara göre hat genişlikleri ve uzunlukları hesaplanmış ve Tablo 6'da değerler verilmiştir.

Tablo 6. $n=4$ için empedansların hat genişlik ve uzunluk değerleri

\begin{tabular}{|l|l|l|}
\hline Hat/Hatlar & $\mathbf{W}(\mathbf{m m})$ & $\mathbf{\Lambda}_{\mathbf{g} 0} / \mathbf{4}(\mathbf{m m})$ \\
\hline$Z_{A}, Z_{B}$ & 2.99 & 16.6 \\
\hline$Z_{1}, Z_{4}$ & 0.31 & 18.15 \\
\hline$Z_{2}, Z_{3}$ & 1.62 & 17.2 \\
\hline$Z_{1,2}, Z_{3,4}$ & 2.1 & 17 \\
\hline$Z_{2,3}$ & 1.95 & 17.2 \\
\hline
\end{tabular}

Daha sonra bu değerlere göre devre tasarlanmıştır. Devrenin şematiği Şekil 8'deki gibidir. S-parametre değeri Şekil 9'da gösterilmiştir. 


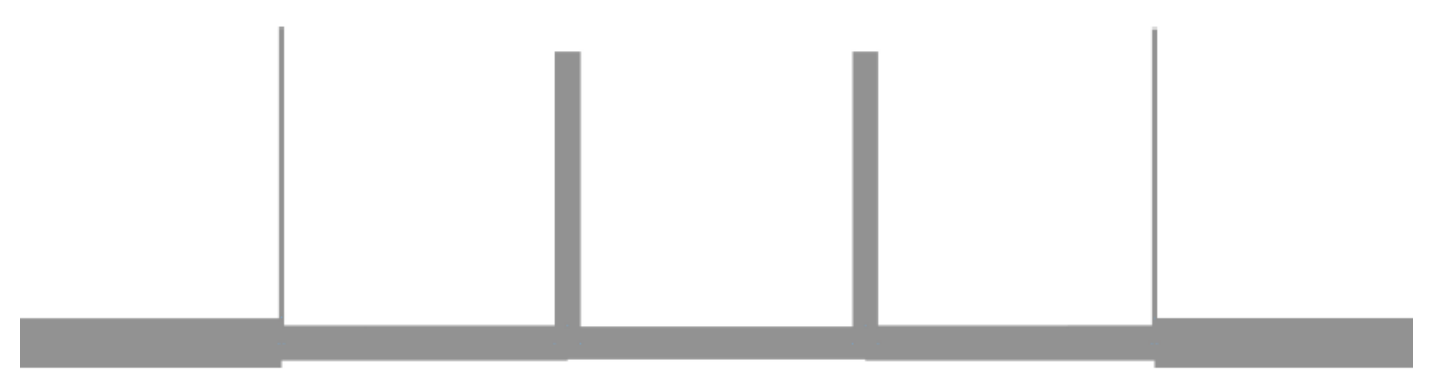

Şekil 8. Optimum bant durduran filtre tasarımı ( $n=4$ için)

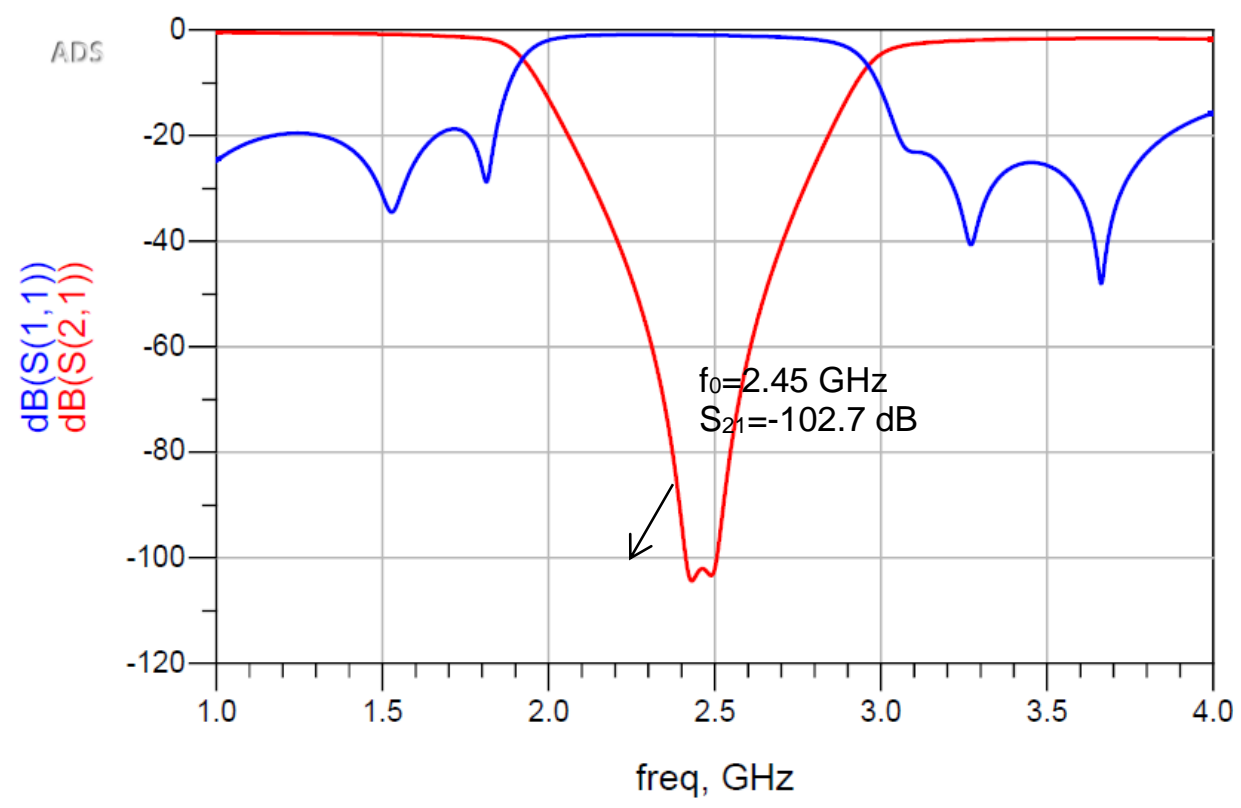

Şekil 9. Optimum bant durduran filtre $S_{11}$ ve $S_{21}$ değeri simülasyon sonucu ( $n=4$ için)

Tasarımı yapılan devrede merkez frekansı $2.45 \mathrm{GHz}$ 'de $\mathrm{S}_{21}$ değeri -102.7 dB olarak ölçülmüştür. Tasarıma ait yüzde bant genişliği eşitlik (10)'dan yararlanılarak \%48.4 olarak hesaplanmıştır.

\section{SONUÇLAR}

Bu çalışmada, 2,45 $\mathrm{GHz}$ merkez frekansına sahip 3 farklı optimum bant durdurucu filtre tasarımı yapılmıştır. Filtre tasarımılarında en yaygın yaklaşımlardan chebyshev kullanılarak, mikroşerit bir hat üzerine eklenen açık devre saplamalar ile optimum bant durduran filtre tasarımları yapılmıştır. Bunlar iki, üç ve dört adet saplama sayısı ile gerçeklenmiştir. Tasarım yapılırken mikrodalga devre analizi yapabilen bir bilgisayar destekli tasarım aracı (ADS) kullanılarak saçılma parametreleri, merkez frekansları ve tasarıma ait uzunluk bilgilerine ulaşılmıştır. S-parametrelerinden yararlanarak tasarımlara ait yüzde bant genişlikleri hesaplanmıştır. Hesaplanan bütün değerler Tablo 7'de özet olarak verilmiştir.
Tablo 7. Tasarımlara Ait Sonuçlar

\begin{tabular}{|l|l|l|l|}
\hline & $\mathbf{n = 2}$ & $\mathbf{n = 3}$ & $\mathbf{n}=\mathbf{4}$ \\
\hline $\mathbf{S}_{21}(\mathbf{d B})$ & -49 & -86.2 & -102.7 \\
\hline $\mathbf{S}_{11}(\mathbf{d B})$ & -1.01 & -0.96 & -0.93 \\
\hline Bant Genişliği (\%) & 29.4 & 41.6 & 48.4 \\
\hline Uzunluk (mm) & 50 & 69 & 88 \\
\hline $\begin{array}{l}\mathbf{f}_{\mathbf{0}} \text { Merkez Frekansı } \\
\text { (GHz) }\end{array}$ & 2,45 & 2,45 & 2,45 \\
\hline
\end{tabular}

Tablo 7'de verilen tasarımlara ait sonuçlar incelendiğinde; ölçülen yüzde bant genişlikleri saplama sayısı (n) arttıkça tasarım yapılmadan önce seçilen kısmi bant genişliğine (KBG) yakınsamıştır. Tasarımlarda saplama sayısı arttıkça $S_{21}$ araya girme kaybı (insertion loss) değerinde daha iyi sonuçlar elde edilmiş olup buna paralel olarak $S_{11}$ geri yansıma kaybı (return loss) 0'a yakınsamıştır. Bu sonuçlar bant durduran filtrelerde olması beklenen değerlerdir. Tasarımları yapılmış olan filtrelerdeki en büyük avantaj bant genişliği dahilinde araya girme kaybının $\left(S_{21}\right)$ ve geri yansıma kaybının $\left(S_{11}\right)$ ideal'e yaklaşmasıdır. Saplama sayısı arttıkça yüzde bant genişliği 
artmıştır. Fakat kullanım yeri açısından mikrodalga devre tasarımlarında devre boyutun önemli büyüktür. Saplama sayısının artması ile birlikte tasarım, mikrodalga devrelerde olması istenen kompakt boyuttan uzaklaşmıştır. Kullanılacak yere, istenilen bant genişliğine ve istenilen performansa göre tasarım parametreleri belirlenebilir.

Filtre tasarımılarında ideal filtreye en yakın tasarımın gerçeklenmesi hedeflenir. Bunun için de geliştirilmiş farklı yaklaşım metodları vardır. Bu çalışma ile chebyshev yaklaşım metodu kullanılarak optimum bant durduran filtre tasarımları yapılmış ve sonuçlar beklenen değerlere yakın olarak şekillenmiştir. Tasarım yapılırken mikrodalga devre analizi yapabilen bir bilgisayar destekli tasarım aracı olan ADS kullanılarak tasarım parametreleri bu program üzerinden ölçülmüştür.

\section{KAYNAKLAR}

BalaSenthilMurugan, L., Antony Anbu Raja, S., Deeban Chakravarthy, S., Kanniyappan, N. (2012). Design and Implementation of a Microstrip Band-Stop Filter for Microwave Applications, International Conference on Modelling Optimisation and Computing, 10-11 April 2012,Kumarakoil, INDIA, 1346-1351.
Habibi, R., Ghobadi, Ch., Nourinia, J., Ojaroudi, M., Ojaroudi, N. (2012). Very compact broad band-stop filter using periodic L-shaped stubs based on self-complementary structure for X-band application, Electronics Letters, 48(23):1481-1482

Hong, J., Lancaster, M. J. (2001). Microstrip Filters for RF/Microwave Applications, John Wiley \& Sons, New York.

Hsieh, M., Wang, S. (2005). Compact and Wideband Microstrip Bandstop Filter, IEEE Microwave And Wireless Components Letters, Vol. 15, No. 7, 472-474

Kusama, Y., Isozaki, R. (2019). Compact and Broadband Microstrip Band-Stop Filters with Single Rectangular Stubs. Applied Sciences MDPI Journals, 9(2): 248

Liu, G., Xu, J., Liu, Z. (2017). A Narrowband Absorptive BandStop Filter Based on a Resistor-Loaded Compact Resonator. 2017 Progress in Electromagnetics Research Symposium - Spring (PIERS), 22-25 May, St. Petersburg, Russia

Shaman, H., Hong, J. (2007). Wideband Bandstop Filter with Cross-Coupling, IEEE Transactions on Microwave Theory and Techniques, 55( 8):1780-1785.

Thede, L. (2004). Practical Analog and Digital Filter Design ,Artech House Inc., Ohio.

Tu, W., Chang, K., (2006). Compact Second Harmonic-Suppressed Bandstop and Bandpass Filters Using Open Stubs, IEEE Transactions on Microwave Theory and Techniques, 54(6): 2497-2501

Yang, S. (2015). A Dual-Band Bandstop Filter Having Open Stubs and Two Equivalent T-Shaped Lines. International Journal of Electromagnetics and Applications, 5(3): 108111. 